\title{
LETTERS
}

The purpose of this Letters section is to provide rapid dissemination of important new results in the fields reg ularly covered by Physics of Fluids A. Results of extended research should not be presented as a series of letters in place of comprehensive articles. Letters cannot exceed three printed pages in length, including space allowed for title, figures, tables, references and an abstract limited to about 100 words. There is a three-month time limit, from date of receipt to acceptance, for processing Letter man uscripts. Authors must also submit a brief statement justifying rapid publication in the Letters section.

\section{Flame length measurements of the turbulent plume}

\author{
H. Johari \\ Department of Mechanical Engineering, Worcester Polytechnic Institute, Worcester, Massachusetts 01609
}

(Received 21 February 1990; accepted 30 March 1990)

The minimum molecular scale mixing rate of the turbulent plume was found by measurements of the plume "flame length" under various initial conditions. A simple derivation based on the self-similar properties of plumes provides power law relations for the mixing rate, which agree well with the experiments.

A steady source of buoyancy creates a plume. Experimental and theoretical studies of plumes have been mainly concerned with the far-field (self-similar) velocity and density profiles. ${ }^{1-3}$ Yet in many practical situations, knowledge regarding the rate of molecular scale mixing of the buoyant fluid with its environment is needed. This is especially true in the context of atmospheric studies, where models for the formation and growth stages of clouds utilize the turbulent plume dynamics. In this Letter a simple argument for mixing in plumes is presented and its implications are examined in light of the experimental findings.

A global measure of plume entrainment $E$ is the ratio of axial volume flux $Q$ at any station to the initial volume flux $Q_{0}$ at the source. Rouse, Yih, and Humphreys' reported the mean plume volume flux in the self-similar region to be

$$
Q=0.153 F^{1 / 3} z^{5 / 3} \text {, }
$$

where $F$ is the buoyancy flux (conserved in an unstratified environment) and $z$ is the distance from the plume source (virtual origin). The source volume flux $Q_{0}$ in terms of the nozzle exit (average) velocity $w_{0}$ and diameter $d$ is

$$
Q_{0}=\left(\pi d^{2} / 4\right) w_{0} \text {. }
$$

In Eq. (1), $F$ may be replaced with the source buoyancy flux $F_{0}$ in cases where the ambient fluid is not stratified, where

$$
F_{0}=\left(\pi d^{2} / 4\right) w_{0} g\left[\left(\rho_{0}-\rho_{a}\right) / \rho_{a}\right] .
$$

Here, $g$ is the gravitational acceleration and $\rho_{0}$ and $\rho_{a}$ are nozzle and ambient fluid densities. The entrainment parameter $E$ can be written as

$$
E=Q / Q_{0}=0.18 \mathrm{Ri}_{0}^{1 / 3}(z / d)^{5 / 3},
$$

where $\mathbf{R i}_{0}$ is the nozzle Richardson number defined as

$$
\mathrm{Ri}_{0}=g\left[\left(\rho_{0}-\rho_{a}\right) / \rho_{a}\right] d w_{0}^{-2} \text {. }
$$

On the other hand, Broadwell and Breidenthal ${ }^{4}$ argue that for large Reynolds number free shear flows of high Schmidt number $(\mathrm{Sc} \equiv v / D$, where $v$ and $D$ are the kinematic viscosity and diffusion coefficient) fluids, the mixing is controlled by the entrainment rate of large eddies. Then, for an entrainment-limited mixing process, Eq. (2) may be used to answer the following question: What is the minimum distance from a source to have every released buoyant fluid parcel mix with the ambient fluid to a prescribed volumetric ratio $\phi$ ? This distance will be referred to as the flame length $L$, associated with the ratio $\phi$. In fact, a lower bound for the molecular scale mixing rate may be established by measuring the flame length for various values of $\phi .^{5}$

Broadwell $^{5}$ developed a simple dilution argument for jets. Following him, the argument is derived for buoyancydriven plumes. In a time duration $\Delta t$, the volumetric ratio of entrained fluid to released plume fluid is given by $E$,

$$
E=\frac{Q \Delta t}{Q_{0} \Delta t}=\frac{\left(Q_{0}+Q_{e}\right) \Delta t}{Q_{0} \Delta t}=\frac{Q_{e} \Delta t}{Q_{0} \Delta t}+1,
$$

where $Q_{e}$ is the volume flow rate of the entrained fluid. The volumetric mixing ratio $\phi$ is then proportional to $\left(Q_{e} \Delta t\right) /\left(Q_{0} \Delta t\right)$ for an entrainment-limited mixing process. Hence

$$
1+\phi \sim 1+\left(Q_{e} \Delta t\right) /\left(Q_{0} \Delta t\right)=E .
$$

An expression for the flame length is found by replacing $L$ for $z$ and $E$ in terms of $\phi$ in Eq. (2) to obtain

$$
(1+\phi) \sim \mathrm{Ri}_{0}^{1 / 3}(L / d)^{5 / 3}
$$

or

$$
L / d=C_{m}(1+\phi)^{3 / 5} \mathrm{Ri}_{0}^{-1 / 5} .
$$

The mixing coefficient $C_{m}$ is a proportionality constant and must be found from experiments.

A series of experiments were performed in a Lucite water tank of $1.7 \mathrm{~m}^{3}$ volume. The plume fluid (brine solutions of various densities) was released into the ambient fluid (fresh water) near the top of the tank through the combination of a calibrated valve and a nozzle. Three different nozzle exit diameters $(0.48,0.95$, and $1.43 \mathrm{~cm})$ were utilized. The tank and plume fluids contained small concentrations 


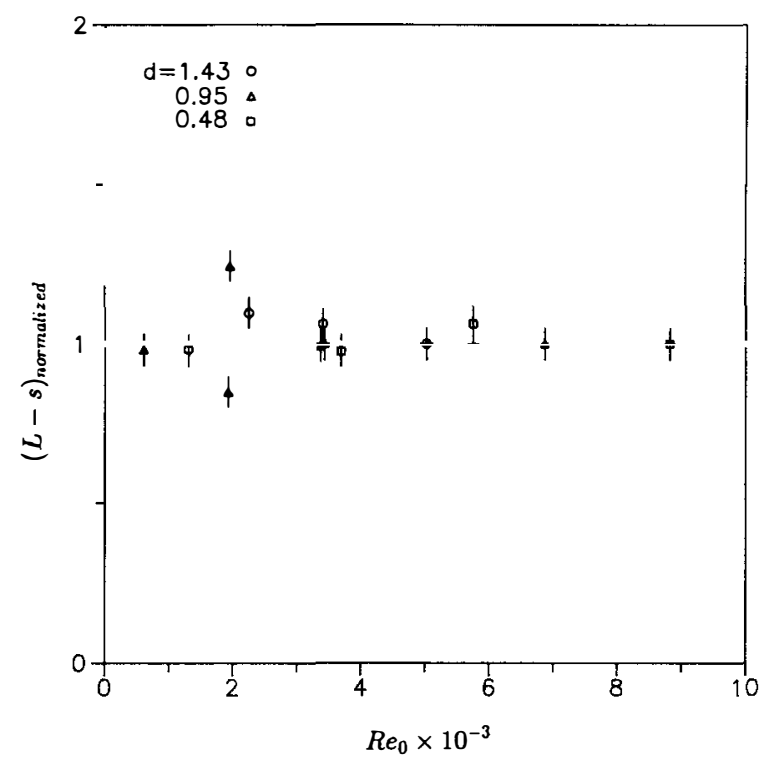

FIG. 1. Effect of nozzle Reynolds number on normalized plume flame length. Error estimates are typically $\pm 5 \%$.

(about $10^{-3} \mathrm{M}$ ) of acid $\left(\mathrm{H}_{2} \mathrm{SO}_{4}\right)$ and base $(\mathrm{NaOH})$, respectively. The presence of phenolphthalein, a $p \mathrm{H}$ indicator, in the released fluid rendered it a purplish color. However, upon mixing with the ambient fluid to a prescribed volumetric ratio $\phi$, the indicator disappeared. By photographing the plume, the location of the last parcel to discolor was determined. The distance from this point to the nozzle was termed "flame length," and hereafter will be scaled with the nozzle diameter. The acid-base chemistry is fast compared with the fluid mechanical mixing. This technique has been previously used to measure the flame length of momentum-driven turbulent jets. $^{6.7}$

Prior experiments on turbulent jets ${ }^{6.7}$ had shown that for nozzle Reynolds numbers beyond a value of 3000 , the jet flame length becomes independent of the Reynolds number. In order to see if a similar effect exists in turbulent plumes, a series of runs were made in which the nozzle Reynolds number $\mathrm{Re}_{0}$ was varied systematically while the nozzle Richardson number and $\phi$ were held constant. Although the plume Reynolds number (based on the local flow diameter and centerline velocity) increases in the downstream direction, the nozzle Reynolds number sets a minimum value for the entire flow field.

Normalized flame length measurements of plumes with various initial conditions are plotted against the nozzle Reynolds number in Fig. 1. The breakpoint distance $s$, the height from the nozzle exit where the plume starts to spread, is subtracted from flame length $L$ to be in conformity with other investigators. ${ }^{6.8}$ Since the "flame" tip fluctuated continuously, each flame length data point is an average value for the run. Furthermore, in this plot each measurement is normalized by the flame length at the largest (nozzle) Reynolds number, for the same $\phi$ and $\mathrm{Ri}_{0}$. Data points with nozzle Reynolds numbers greater than 3500 fall on a horizontal line (to within the experimental error), indicating that the flame length is independent of Reynolds number.

To verify the power law dependence of flame length on

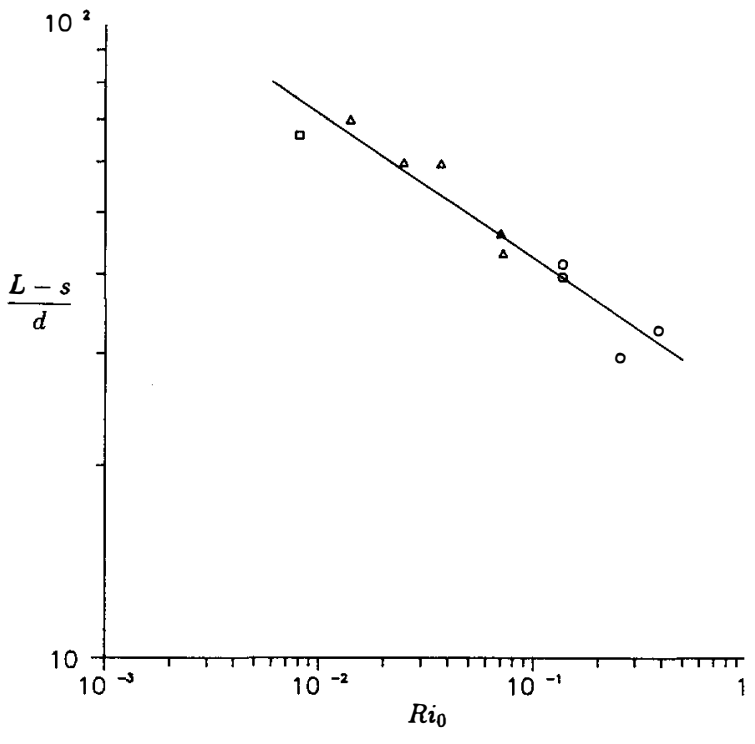

FIG. 2. Logarithmic plot of plume flame length as a function of nozzle Richardson number; $\operatorname{Re}_{0}=3500$ and $\phi=12$. The line has a slope of $-\frac{1}{\xi}$.

the nozzle Richardson number, a series of runs were made in which $\operatorname{Re}_{0}(=3500)$ and $\phi(=12)$ were held constant. Figure 2 is a logarithmic plot of $(L-s) / d \mathrm{vs} \mathrm{Ri}_{0}$. Through the use of different initial buoyancies and three nozzle exit diameters, the nozzle Richardson number was varied by a factor of about 50. The line in Fig. 2, drawn according to Eq. (5), has a slope of $-\frac{1}{5}$. The measured flame lengths and the power law relationship for the initial Richardson number appear to agree well.

The next step is to check if the power law in Eq. (5) holds over an extended range of volumetric mixing ratios $\phi$ and to find the value of the plume mixing constant $C_{m}$. A large number of experiments were done with $\phi$ ranging from less than 1 to more than 35 and a variety of initial Richard-

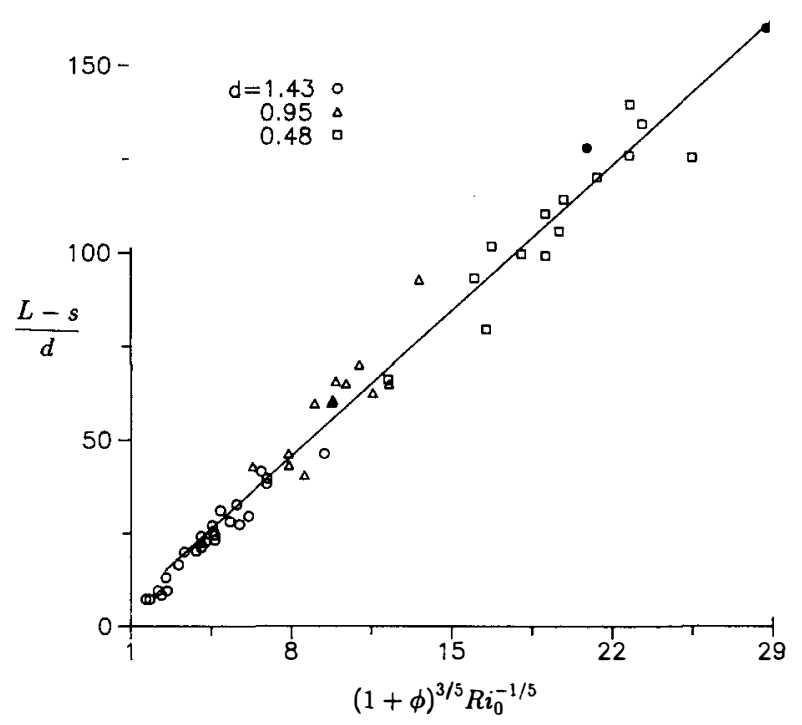

FIG. 3. Dependence of plume flame length on $(1+\phi)^{3 / 5} \mathrm{Ri}_{0}{ }^{1 / 5}$. The filled symbols are propane flame length data from Ref. 9. 


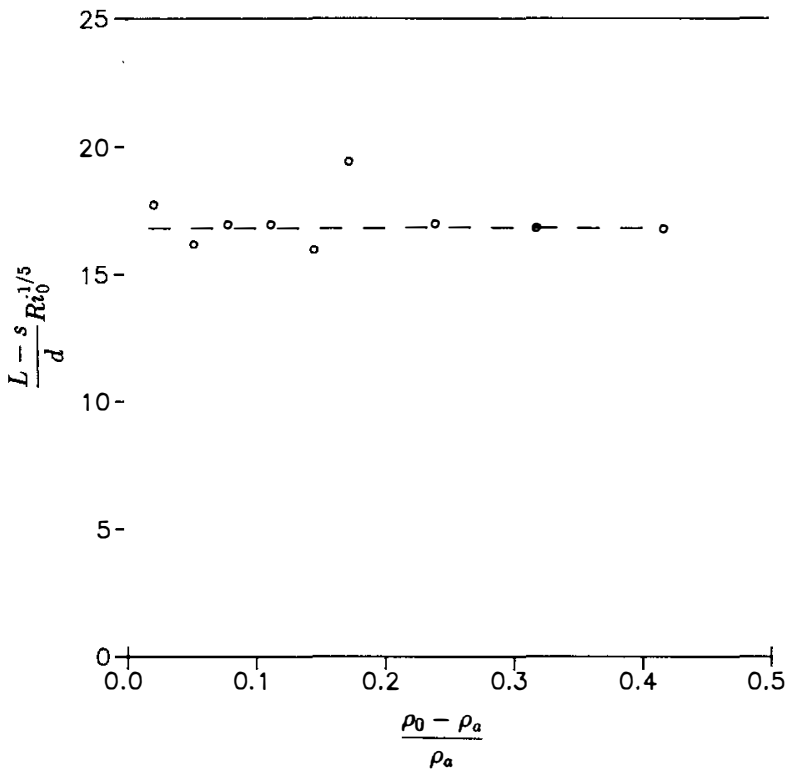

FIG. 4. Effect of initial density difference on plume flame length; $\operatorname{Re}_{i)}=5000$ and $\phi=6$. The dashed line represents the average value of the plotted data.

son numbers. A minimum nozzle Reynolds number of 3500 was maintained in all cases. The flame length $(L-s) / d$ as a function of $(1+\phi)^{3 / 5} \mathrm{Ri}_{0}^{-1 / 5}$ is plotted in Fig. 3. There is good agreement between the experimental data and the straight line representing Eq. (5), with $C_{m}$ chosen as 5.5. This value is obtained through a least-squares fit to all of the data points, except the ones with very small abscissa values. There are also two data points from propane combustion studies of Becker and Yamazaki, ${ }^{9}$ where the flame tip was in the buoyancy-driven regime. The combustion data points also appear to follow the scaling laws proposed in Eq. (5).

In a series of experiments, $\phi$ was consistently reduced from 3 to $\frac{1}{3}$ while all the other parameters were kept nearly constant. The flame length decreased continuously until it reached a minimum value of $8 d$. Therefore it was concluded that at least about eight diameters are needed to mix every plume parcel with even small fractions of the tank fluid.

The influence of large initial density differences on molecular scale mixing was investigated in another series of experiments. This was also a test of the Boussinesq approximation and its limitations, since the analysis leading to the flame length power law in Eq. (5) benefited from simplifications of the Boussinesq assumption. In this set of runs $\operatorname{Re}_{0}$ and $\phi$ were fixed at 5000 and 6 , respectively. The initial density difference, divided by the ambient density, was varied from $2 \%$ to nearly $42 \%$. Keeping the nozzle diameter and Reynolds number constant while varying the initial buoyancy created different nozzle Richardson numbers. In order to remove the effects of $\mathrm{Ri}_{0}$, the flame length was divided by $\mathrm{Ri}_{0}^{-1 / 5}$. The parameter $(L-s) \mathrm{Ri}_{0}^{1 / 5} / d$ is plotted as a function of $\left(\rho_{0}-\rho_{a}\right) / \rho_{a}$ in Fig. 4. Results indicate that to first order, mixing remains unaffected by the variations in initial buoyancy after accounting for the Richardson number effects. The local average buoyancy at the flame tip for these runs was never greater than $3.5 \%$; a large local buoyancy there might be required to alter the flame length significantly.

In summary, the flame length of turbulent plumes, scaled by the nozzle diameter, is solely dependent on the volumetric mixing ratio and the nozzle Richardson number, as long as the Reynolds number is kept above 3500 . The measurements verify the validity of a simple derivation based on the self-similar properties of plumes. The assumption of entrainment-limited mixing processes in the turbulent plume appears to be valid.

\section{ACKNOWLEDGMENTS}

The author is indebted to Professor R. E. Breidenthal for his guidance and constant support.

This effort was funded by National Science Foundation Grant No. ATM-8611225.

'H. Rouse, C. S. Yih, and H. W. Humphreys, Tellus 4, 201 (1952).

${ }^{2}$ B. R. Morton, G. I. Taylor, and J. S. Turner, Proc. R. Soc. London Ser. A 234, 1 (1956).

${ }^{3}$ H. B. Fischer, E. J. List, R. C. Koh, J. Imberger, and N. H. Brooks, Mixing in Inland and Coastal Waters (Academic, New York, 1979).

${ }^{4}$ J. E. Broadwell and R. E. Breidenthal, J. Fluid Mech. 125, 397 (1982).

${ }^{5} \mathrm{~J}$. E. Broadwell, Turbulent Reactive Flows: USA-France Workshop on Turbulent Reactive Flows, Lecture Notes in Engineering, edited by R. Borghi and S. N. B. Murthy (Springer, New York, 1987), Vol. 40, p. 257.

'D. S. Weddell, Sc.D. dissertation, Massachusetts Institute of Technology, 1941.

W. J. A. Dahm and P. E. Dimotakis, AIAA J. 25, 1216 (1987).

${ }^{x}$ W. R. Hawthorne, D. S. Weddell, and H. C. Hottel, 3rd Symposium on Combustion and Flame and Explosion Phenomena (Williams and Wilkins, Baltimore, MD, 1948)

${ }^{9}$ H. A. Becker and S. Yamazaki, Combust. Flame 33, 123 (1978). 